ISSN 0103-5150

Fisioter. Mov., Curitiba, v. 26, n. 3, p. 689-695, jul./set. 2013

Licenciado sob uma Licença Creative Commons

\title{
Comportamento da frequência cardíaca em imersão nas situações de repouso e durante exercícios de hidroginástica
}

\author{
Heart rate behavior in immersion in cases \\ of rest and during water exercises
}

\section{Mabel Micheline Olkoski ${ }^{[a]}$, Adair da Silva Lopes ${ }^{[b]}$}

[a] Mestre em Treino em Alto Rendimento pela Universidade Técnica de Lisboa, professora da área de Ciências da Saúde da Universidade Comunitária da Região de Chapecó (Unochapecó), Chapecó, SC - Brasil, e-mail: mabelolkoski@unochapeco.edu.br

[b] Doutor em Educação Física pela Universdade Federal de Santa Maria (UFSM), professor do Centro de Desportos da Universidade Federal de Santa Catarina (UFSC), Florianópolis, SC - Brasil, e-mail: adair.lopes@ufsc.br

\section{Resumo}

Introdução: A frequência cardíaca é um parâmetro de fácil utilização para prescrição e controle da intensidade do exercício. Ao realizar um exercício no meio aquático ocorrem alterações fisiológicas significativas que devem ser compreendidas para a prescrição de exercícios, ocasionando assim os efeitos adaptativos adequados. Objetivo: Realizar uma revisão sistemática da literatura sobre o comportamento da frequência cardíaca em imersão nas situações de repouso e durante exercícios de hidroginástica e sobre os fatores que a influenciam. Resultados: Os estudos envidenciam que a frequência cardíaca pode ser influenciada pela profundidade devido ao gradiente de pressão hidrostática exercido sobre o corpo, bem como à termorregulação diferenciada. Em repouso, a frequência cardíaca pode variar de acordo com as diferentes temperaturas da água, posições corporais e profundidades. No entanto, a magnitude do efeito de cada um desses fatores sobre a frequência cardíaca ainda não é bem definido. Conclusão: Durante a realização de exercícios de hidroginástica, essa variável é proporcional à velocidade de execução do exercício, à utilização de equipamento resistivo, à área projetada e ao grupo muscular envolvido e inversamente proporcional à profundidade.

Palavras-chave: Frequência cardíaca. Imersão. Hidroginástica. 
Abstract

Introduction: Heart rate is an easy parameter to control and prescribe exercise intensity. When performing an exercise in aquatic environment significant physiological changes occur; these changes must be understood to prescribe exercise, causing to appropriate adaptive effects. Objective: To conduct a systematic review of literature on the behavior of heart rate by immersion in rest situations and during aquatics exercises, as well as understanding the factors that influence it. Results: The results showed that heart rate may be influenced by the immersion due to the gradient of hydrostatic pressure exerted on the body and to different thermoregulation. At rest, the heart rate can change according to different water temperatures, body positions and depths of immersion. However, the magnitude of the effect of each factor on the heart rate has not been well defined. Conclusion: During aquatic exercise, this variable is proportional to the speed of execution, use of resistive equipment, projected area and the muscle group involved and inversely proportional to the depth of immersion.

Keywords: Heart rate. Immersion. Aquatic exercise.

\section{Introdução}

A hidroginástica é um exercício físico que, além de utilizar equipamentos resistivos, aproveita a resistência da água como sobrecarga (1) durante uma sessão em que os movimentos são geralmente realizados em posição ortostática. Pesquisas têm verificado a melhora da condição física de adultos, idosos (2) e estudantes universitários, tanto do sexo masculino (3) quanto do feminino (4). Além de propiciar benefícios à forma física, o exercício realizado no meio líquido causa menor impacto nos membros inferiores, possibilitando a prática de exercício físico a pessoas que, fora da água, não podem suportar o seu próprio peso ao realizarem um exercício (1).

A aferição da frequência cardíaca ( $\mathrm{FC}$ ) tem sido bastante utilizada para a prescrição e controle da intensidade do exercício físico por ser um parâmetro de fácil obtenção, baixo custo e associação com variáveis fidedignas de mensuração de esforço no meio terrestre (5).

Mas quando os indivíduos entram na água, sofrem alterações fisiológicas diferentes daquelas verificadas no meio terrestre $(6,7)$. Apesar de ser um parâmetro de fácil obtenção, a FC, tanto em repouso quanto durante o exercício no meio aquático, é um dos parâmetros influenciados pela pressão hidrostática (8) e pela termorregulação $(9,10)$. Assim, o objetivo deste estudo foi realizar uma revisão sistemática da literatura sobre o comportamento da FC em imersão nas situações de repouso e durante exercícios de hidroginástica e sobre os fatores que a influenciam.

\section{Mareriais e métodos}

Para o desenvolvimento desta revisão, foram obtidas referências clássicas e atuais nas bases de dados PubMed, SPORTDiscus e SciELO durante os meses de novembro e dezembro de 2010. Para tal, foram utilizados os seguintes descritores em língua portuguesa na base de dados SciELO: "frequência cardíaca", "exercícios aquáticos", "imersão", "repouso", "hidroginástica". Nas bases de dados PubMed e SPORTDiscus utilizou-se: "heart rate", "aquatic exercise", "imersion", "rest situation", inseridos valendo-se de comandos OR e AND (e suas respectivas traduções para a base SciELO).

Com o intuito de efetuar uma busca específica dos estudos, foram utilizados alguns critérios para seleção dos artigos. Os critérios de inclusão adotados foram: avaliação do artigo por pares, trabalhos atuais e clássicos nas línguas portuguesa e inglesa, realizados com seres humanos de ambos os sexos e todas as faixas etárias. Os critérios de exclusão foram: teses, dissertações, trabalhos em que eram disponibilizados apenas o resumo e os que apresentavam falhas metodológicas.

O procedimento foi organizado em três etapas: (i) levantamento de todos os artigos encontrados a partir da procura dos descritores em cada base de dados; (ii) leitura dos títulos e resumos de todos os 
artigos; e (iii) leitura completa dos artigos que atendiam aos critérios de inclusão do estudo.

\section{Resultados e discussão}

\section{Meio aquático e a $\mathrm{FC}$ de repouso $(\mathrm{FCr})$}

Nas bases de dados pesquisadas foram encontrados 19 artigos que avaliaram o comportamento da frequência cardíaca de repouso (FCr) em diferentes profundidades de imersão. Entre eles, dez atenderam aos critérios de inclusão. Os artigos selecionados para leitura e análise são especificados no Quadro 1:

Os estudos apresentaram resultados controversos a respeito do comportamento da $\mathrm{FCr}$ no meio líquido. Enquanto alguns sugerem a ocorrência de uma bradicardia $(7,8,11,12)$, o trabalho de Arborelius et al. (13) sustenta a tese de que não existe alteração da FCr com a imersão.

Watenpaugh et al. (8) verificaram bradicardia tanto em mulheres quanto em homens ao imergirem até a profundidade do pescoço em água termoneutra $\left(35^{\circ} \mathrm{C}\right.$ em repouso). Kruel et al. (11) avaliaram apenas homens jovens e também verificaram uma diminuição da FCr quando os sujeitos foram imersos até a profundidade da cicatriz umbilical. Mas, nesse trabalho, a temperatura da água permaneceu abaixo da termoneutra $\left(31^{\circ} \mathrm{C}\right)$. Para temperaturas semelhantes $\left(30^{\circ} \mathrm{C}\right)$, Craig e Dvorak (12) também verificaram diminuição dos valores de $\mathrm{FCr}$ na profundidade da linha do queixo.

Essa diminuição da FCr com a imersão, mesmo com alterações da tempratura da água, possivelmente se deve à pressão hidrostática existente nesse meio, que provoca uma hipervolemia central proporcionando alterações fisiológicas na $\mathrm{FCr}$ (8). Com a imersão do corpo em órtese na água, o gradiente hidrostático de pressão exercido na superfície do corpo favorece a estimulação de barorreceptores periféricos, os quais auxiliam na redistribuição do sangue das extremidades inferiores e abdome para a região torácica, aumentando o volume sanguíneo central (13) e, consequentemente, diminuindo a FCr. Além disso, essa bradicardia pode ser explicada pela acentuada dissipação de calor na água (12).

Ao analisar os trabalhos que tiveram por objetivo examinar o comportamento da FCr do sujeito em órtese no meio líquido em temperatura termoneutra (14), um estudo não encontrou alterações dessa variável (13). Arborelius et al. (13) não observaram alterações ao avaliarem homens imersos até o pescoço.
Esses resultados seriam os esperados, uma vez que a temperatura termoneutra seria aquela que não fosse capaz de provocar alterações fisiológicas em repouso (14).

Muitos estudos foram desenvolvidos com o intuito de apurar os possíveis efeitos das diferentes temperaturas de água sobre a $\operatorname{FCr}(9,10,12,15,16)$. De forma geral, nota-se que em temperaturas de água maior ou igual a $36^{\circ} \mathrm{C}$ (considerada termoneutra na situação repouso) a $\mathrm{FCr}$ aumenta ou não sofre alterações. Já em temperaturas menores do que $34^{\circ} \mathrm{C}$, há diminuição da $\mathrm{FCr}$, com exceção de temperaturas muito baixas (9), $14{ }^{\circ} \mathrm{C}$, por exemplo, podendo ocorrer taquicardia. Nesse caso, constata-se que a temperatura corporal aumenta cerca de $1,7^{\circ} \mathrm{C}$ induzindo à estimulação termogênica e ativação do sistema nervoso simpático (evidente a partir da maior concentração de noradrenalina). Esse aumento da noradrenalina provoca elevação da $\mathrm{FCr}$, quando comparado aos indivíduos imersos em temperatura de água termoneutra.

Esses resultados que indicam comportamento proporcional entre a $\mathrm{FCr}$ e a temperatura da água podem justificar os achados dos trabalhos que avaliaram as respostas de $\mathrm{FCr}$ abaixo da termoneutra $(11,12)$ e encontraram diminuições da FCr.

Com a leitura dos trabalhos, observou-se que a mudança da posição corporal também vem sendo considerada um dos fatores que ifluenciam a variável em questão $(11,17)$.

Sheldahl et al. (17) não encontraram diferenças ao observar as respostas de FCr em homens nas posições de decúbito dorsal e otostática no ambiente terrestre e aquático $\left(31^{\circ} \mathrm{C}\right)$. Kruel et al. (11) analisaram as respostas da FCr de homens imersos na posição ortostática até a profundidade da cicatriz umbilical, em temperatura de $30^{\circ} \mathrm{C}$, comparando com as posições decúbito dorsal e ortostática fora da água. A FCr foi menor quando os sujeitos permaneceram imersos e durante a posição decúbito dorsal fora da água. Segundo os autores, essas duas posições (ortostática dentro da água e decúbito dorsal fora da água) apresentaram menores valores e semelhanças devido ao retorno venoso facilitado pela posição corporal (decúbito dorsal fora da água) e pela pressão hidrostática (ortostática dentro da água). Mas esses estudiosos afirmaram que a temperatura da água abaixo da termoneutra $\left(30^{\circ} \mathrm{C}\right)$ possa ter influenciado esses resultados, conforme explicado anteriormente.

A profundidade na qual o indivíduo permanece parece ser outro fator que influencia a FCr. Kruel et al. (18) avaliaram 395 indivíduos de ambos os sexos 
Olkoski MM, Lopes AS.

Quadro 1 - Síntese dos resultados dos artigos que analisaram o comportamento da frequência cardíaca de indivíduos em imersão na situação de repouso

\begin{tabular}{|c|c|c|c|}
\hline Autor/ano & Objetivo & Amostra & Principais conclusões \\
\hline Craig \& Dvorak (12) & $\begin{array}{l}\text { Verificar as alterações fisiológicas } \\
\text { durante a imersão na água em } \\
\text { diferentes temperaturas. }\end{array}$ & $\begin{array}{l}\text { Dez sujeitos jovens e } \\
\text { saudáveis. }\end{array}$ & $\begin{array}{l}\text { FCr maior em temperaturas } \\
\text { acima da termoneutra. FCr } \\
\text { menor em temperaturas } \\
\text { abaixo da temoneutra. }\end{array}$ \\
\hline Rennie et al. (15) & $\begin{array}{l}\text { Averiguar as respostas } \\
\text { fisiológicas durante a imersão em } \\
\text { repouso durante vários tempos e } \\
\text { temperaturas da água. }\end{array}$ & Sujeitos jovens. & $\begin{array}{l}\text { Sem alterações da FCr em } \\
\text { temperatura termoneutra. } \\
\text { FCr menor em temperaturas } \\
\text { abaixo da termoneutra. }\end{array}$ \\
\hline Arborelius et al. (13) & $\begin{array}{l}\text { Avaliar o rendimento cardíaco } \\
\text { durante a posição sentado fora da } \\
\text { água e em órtese, imersos até os } \\
\text { ombros. }\end{array}$ & $\begin{array}{l}\text { Dez sujeitos suecos ( } 24 \\
\text { anos). }\end{array}$ & $\begin{array}{l}\text { Sem alterações da FCr nas } \\
\text { duas situações. }\end{array}$ \\
\hline Sheldahl et al. (17) & $\begin{array}{l}\text { Investigar os efeitos de diferentes } \\
\text { níveis de volume de sangue } \\
\text { central sobre o desempenho } \\
\text { cardíaco em diferentes posições } \\
\text { corporais. }\end{array}$ & Homens jovens. & $\begin{array}{l}\text { Sem alterações da FCr nas } \\
\text { diferentes posições corporais. }\end{array}$ \\
\hline Park et al. (16) & $\begin{array}{l}\text { Determinar as respostas } \\
\text { fisiológicas com a imersão na } \\
\text { água em temperatura termoneutra } \\
\text { e fria. }\end{array}$ & $\begin{array}{l}\text { Dez homens }(22,6 \pm 0,4 \\
\text { anos), sem histórico de } \\
\text { doença cardiovascular. }\end{array}$ & $\begin{array}{l}\text { FCr menor em temperatura } \\
\text { fria. Sem alterações em } \\
\text { temperatura termoneutra. }\end{array}$ \\
\hline Watenpaugh et al. (8) & $\begin{array}{l}\text { Analisar as respostas } \\
\text { cardiovasculares de homens e } \\
\text { mulheres em imersão. }\end{array}$ & $\begin{array}{l}\text { Nove homens (19-28 anos) } \\
\text { e dez mulheres (20-27 anos) } \\
\text { dinamarqueses, saudáveis. }\end{array}$ & $\begin{array}{l}\text { FCr menor durante a imersão } \\
\text { em ambos os sexos. }\end{array}$ \\
\hline Srámek et al. (9) & $\begin{array}{l}\text { Investigar a resposta } \\
\text { cardiovascular de homens } \\
\text { imersos até os ombros em } \\
\text { diferentes temperaturas de água. }\end{array}$ & $\begin{array}{l}\text { Dez homens }(22,2 \pm 2,4 \\
\text { anos), checos, nadadores. }\end{array}$ & $\begin{array}{l}\text { FCr menor quando imersos a } \\
\text { temperatura de } 32^{\circ} \mathrm{C}, 20^{\circ} \mathrm{C} \text { e } \\
\text { FCr maior a } 14^{\circ} \mathrm{C} .\end{array}$ \\
\hline Kruel et al. (18) & $\begin{array}{l}\text { Checar o comportamento da } \\
\text { FCr nos diferentes níveis de } \\
\text { profundidades. }\end{array}$ & $\begin{array}{l}\text { Grupo de } 395 \text { sujeitos, } \\
\text { brasileiros saudáveis e } \\
\text { adaptados ao meio líquido. }\end{array}$ & $\begin{array}{l}\text { FCr proporcional ao nível de } \\
\text { profundidade de imersão. }\end{array}$ \\
\hline Kruel et al. (11) & $\begin{array}{l}\text { Analisar a influência do peso } \\
\text { hidrostático nas mudanças } \\
\text { da FCr em diferentes níveis } \\
\text { de profundidades e posições } \\
\text { corporais. }\end{array}$ & $\begin{array}{l}\text { Dez homens ( } 22,6 \pm 2,12 \\
\text { anos), brasileiros e saudáveis. }\end{array}$ & $\begin{array}{l}\text { FCr menor na posição deitada } \\
\text { e imersão em órtese. }\end{array}$ \\
\hline Graef et al. (10) & $\begin{array}{l}\text { Comportamento da FCr de } \\
\text { indivíduos em órtese imersos } \\
\text { profundidade entre apêndice } \\
\text { xifoide e ombros, em três } \\
\text { temperaturas de água ( } 27,30 \text { e } \\
\left.33^{\circ} \mathrm{C}\right) \text {. }\end{array}$ & $\begin{array}{l}\text { Grupo de } 14 \text { homens (18- } \\
35 \text { anos), praticantes de } \\
\text { atividades aquáticas. }\end{array}$ & $\begin{array}{l}\text { FCr proporcional à } \\
\text { temperatura da água. }\end{array}$ \\
\hline
\end{tabular}

Fonte: Dados da pesquisa.

de 7 a 75 anos. Os sujeitos permaneceram imersos até a profundidade do tornozelo, da cicatriz umbilical e do ombro. Foi detectada diminuição da FCr na análise da profundidade a partir da cicatriz umbilical; ressalta-se que quanto maior a profundidade, maior a bradicardia.
Apesar de alguns resultados contraditórios, os estudos mostram que de uma forma geral a profundidade de imersão influencia a FCr, sendo alterada pelas diferentes posições corporais e temperaturas de água. Os resultados distintos podem ser justificados pelas diferentes metodologias utilizadas, tornando 
difícil determinar a magnitude do efeito de cada um desses fatores sobre a FCr.

\section{FC durante os exercícios de hidroginástica}

Com relação aos trabalhos que investigaram o comportamento da FC durante exercícios de hidroginástica, pôde-se encontrar 44 artigos. Da mesma forma, também foi realizada inicialmente a leitura dos títulos e resumos de todos os artigos, sendo que oito atenderam aos critérios de inclusão. A partir de então, procedeu-se à leitura dos artigos que são apresentados de forma suscinta no Quadro 2.
0 exercício realizado na posição ortostática no meio aquático também sofre influência da pressão hidrostática e da termorregulação. No entanto, essas respostas são contraditórias. Alguns estudos que compararam exercícios de hidroginástica com o mesmo exercício no meio terrestre mostram que a FC é maior durante os exercícios fora da água $(1,19,20)$. Já Johnson et al. (21) encontraram maiores valores de FC durante os exercícios realizados dentro da água. Em todos esses estudos, a velocidade de execussão foi determinada subjetivamente como moderada. Cabe ressaltar que a temperatura da água e a profundidade foram semelhantes nesses trabalhos. Os resultados distintos podem ser justificados pelos

Quadro 2 - Síntese dos resultados dos artigos que analisaram o comportamento da frequência cardíaca durante os exercícios de hidroginástica

\begin{tabular}{|c|c|c|c|}
\hline Autor/ano & Objetivo & Grupo de estudo & Principais conclusões \\
\hline Johnson et al. (21) & $\begin{array}{l}\text { Verificar a FC durante exercícios } \\
\text { realizados dentro e fora da água. }\end{array}$ & $\begin{array}{l}\text { Homens (21-27 anos) e } \\
\text { mulheres ( } 20-29 \text { anos) } \\
\text { noruegueses, estudantes de } \\
\text { Educação Física. }\end{array}$ & $\begin{array}{l}\text { FC maior durante os exercícios } \\
\text { realizados dentro da água. }\end{array}$ \\
\hline $\begin{array}{l}\text { Cassady and Nielsen, } \\
\text { (19) }\end{array}$ & $\begin{array}{l}\text { Avaliar as respostas agudas } \\
\text { de FC durante exercícios } \\
\text { calistênicos de membros } \\
\text { superiores e inferiores, dentro } \\
\text { e fora da água em diferentes } \\
\text { cadências. }\end{array}$ & $\begin{array}{l}\text { Grupo de } 20 \text { homens e } 20 \\
\text { mulheres (19-36 anos), } \\
\text { saudáveis, moderadamente } \\
\text { ativos e não praticavam } \\
\text { exercícios calistênicos. }\end{array}$ & $\begin{array}{l}\text { FC menor durante os exercícios } \\
\text { realizados dentro da água. } \\
\text { FC maior com o aumento da } \\
\text { cadência. }\end{array}$ \\
\hline Alberton et al. (20) & $\begin{array}{l}\text { Examinar a FC durante três } \\
\text { exercícios de hidroginástica } \\
\text { realizados em três cadências, } \\
\text { dentro e fora da água. }\end{array}$ & $\begin{array}{l}\text { Oito mulheres brasileiras, } \\
\text { estudantes de Educação Física. }\end{array}$ & $\begin{array}{l}\text { FC menor durante os exercícios } \\
\text { realizados dentro da água. } \\
\text { FC maior com o aumento da } \\
\text { cadência. }\end{array}$ \\
\hline Kruel et al. (1) & $\begin{array}{l}\text { Observar a FC durante a } \\
\text { realização de cinco exercícios de } \\
\text { hidroginástica em três diferentes } \\
\text { profundidades. }\end{array}$ & $\begin{array}{l}\text { Populaçao de } 23 \text { mulheres ( } 54 \\
\pm 11,16 \text { anos), brasileiras, } \\
\text { praticantes de hidroginástica. }\end{array}$ & $\begin{array}{l}\text { FC menor com o aumento da } \\
\text { profundidade. }\end{array}$ \\
\hline Moraes et al. (23) & $\begin{array}{l}\text { Averiguar o comportamento da } \\
\text { FC durante diferentes exercícios } \\
\text { de hidroginástica. }\end{array}$ & $\begin{array}{l}\text { Grupo de } 65 \text { mulheres } \\
\text { brasileiras, praticantes de } \\
\text { hidroginástica. }\end{array}$ & $\begin{array}{l}\text { FC maior durante os exercícios } \\
\text { com maiores grupos musculares } \\
\text { e áreas projetadas. }\end{array}$ \\
\hline Pinto et al. (24) & $\begin{array}{l}\text { Analisar as respostas de } \\
\text { FC durante um exercício de } \\
\text { hidroginástica realizado com e } \\
\text { sem equipamento resistivo. }\end{array}$ & $\begin{array}{l}\text { Dez mulheres }(21,9 \pm 0,99 \\
\text { anos), brasileiras e praticantes } \\
\text { de hidroginática. }\end{array}$ & $\begin{array}{l}\text { FC maior com utilização de } \\
\text { equipamento resistivo. }\end{array}$ \\
\hline Alberton et al. (22) & $\begin{array}{l}\text { Avaliar a FC durante oito } \\
\text { exercícios de hidroginástica } \\
\text { realizados na mesma cadência. }\end{array}$ & $\begin{array}{l}\text { Oito mulheres brasileiras } \\
(59,63 \pm 4,69 \text { anos), pós- } \\
\text { menopáusicas e praticantes de } \\
\text { hidroginástica. }\end{array}$ & $\begin{array}{l}\text { FC maior durante os exercícios } \\
\text { com maiores grupos musculares } \\
\text { e áreas projetadas. }\end{array}$ \\
\hline Pinto et al. (25) & $\begin{array}{l}\text { investigar as respostas de } \\
\text { FC durante exercícios de } \\
\text { hidroginástica em diferentes } \\
\text { situações. }\end{array}$ & $\begin{array}{l}\text { Grupo de } 11 \text { mulheres brasileiras } \\
\text { entre } 19 \text { e } 26 \text { anos, praticantes } \\
\text { de hidroginástica. }\end{array}$ & $\begin{array}{l}\text { FC maior com utilização do } \\
\text { equipamento resistivo em apenas } \\
\text { um dos membros ou em ambos. }\end{array}$ \\
\hline
\end{tabular}

Fonte: Dados da pesquisa. 
diferentes exercícios utilizados em cada estudo. Isso porque, os diferentes movimentos utilizados (mesmo que realizados numa mesma cadência) provocam diferentes respostas da FC. Alberton et al. (22) estudaram oito exercícios de hidroginástica realizados a uma cadência de $60 \mathrm{bpm}$. Diferentes respostas de FC foram obtidas, e os movimentos com maiores grupos musculares e área projetada envolvida apresentaram maiores valores de FC. Esses achados corroboram com outro trabalho que avaliou diferentes exercícios efetuados no meio aquático (23).

Além do tipo de exercício, a velocidade de execussão do movimento parece ser um fator importante nas respostas da FC. Alberton et al. (20) estudaram três exercícios de hidroginástica praticados dentro e fora da água e em diferentes cadências (80, 100 e 120 bpm). Esses autores encontraram valores maiores de FC conforme o aumento da cadência do exercício. Da mesma forma, Cassady e Nielsen (19) encontraram aumento significativo da FC com o aumento da cadência.

Outro fator importante no aumento da FC é a utilização de equipamentos resistivos. Pinto et al. (24) analisaram a FC durante a realização de dois exercícios executados com e sem equipamento resistivo em mulheres jovens. Os autores verificaram que a FC apresentou maiores respostas com a utilização do equipamento para ambos exercícios. Em outro trabalho, utilizando equipamentos apenas nos membros superiores ou inferiores ou ainda em ambos, verificou-se que a FC foi significativamente maior quando comparada com a não utilização do equipamento (25).

Pode-se verificar que a FC apresenta resultados distintos quando o exercício é realizado dentro e fora da água, chamando a atenção para o fato de o meio aquático provocar alterações fisiológicas. Apesar dessas respostas serem distintas, ressalta-se que alguns fatores influenciam as respostas de FC durante exercícios de hidroginástica, como a velocidade de execussão do movimento (20), o tipo de exercício, a massa muscular envolvida (19,22, 23), a área projetada (26) e a utilização de equipamentos resistivos $(24,25)$.

No que diz respeito à realização de exercícios de hidroginástica em diferentes profundidades, Kruel et al. (1) apuraram que quanto maior a profundidade, menores são as respostas da FC. Segundo esses autores, a diminuição do peso hidrostático e a pressão hidrostática resultam em menor recrutamento muscular e, consequentemente, em menor FC.

Todos os trabalhos encontrados na presente pesquisa, que envolveram exercícios de hidroginástica, foram realizados em temperatura de água termoneutra. Não foram encontrados estudos que avaliavam a influência de diferentes temperaturas de água em exercícios de hidroginástica. Essa lacuna torna-se relevante uma vez que a temperatura da água parece influenciar a FCr.

\section{Conclusão}

A maioria dos estudos mostra que a FC sofre alterações com a imersão no meio aquático tanto em repouso como durante exercícios de hidroginástica. Em repouso, a FC varia de acordo com as diferentes temperaturas da água, posições corporais e profundidades. A magnitude do efeito de cada um desses fatores sobre a FCr não está bem definida, uma vez que os resultados são contraditórios e utilizam diferentes metodologias, não estabelecendo qual dos fatores possui maior influência.

Durante a realização de exercícios de hidroginástica, a FC é proporcional à velocidade de execussão, à utilização de equipamento resistivo, à área projetada e ao grupo muscular envolvido; mas é inversamente proporcional à profundidade.

Dessa forma, confirma-se a necessidade do cuidado do profissional na prescrição ou no controle do exercício realizado no meio líquido a partir da FC, levando-se em consideração as alterações que esse meio provoca.

\section{Referências}

1. Kruel LMF, Moraes EZC, Ávila AOV, Sampedro RMF. Alterações fisiológicas e biomecânicas em indivíduos praticando exercícios de hidroginástica dentro e fora d'água. Kinesis. 2001;(n. especial):104-54.

2. Tsourlou T, Benik A, Dipla K, Zafeiridis A, Kellis S. The effects of a twenty four-week aquatic training program on muscular strenght performance in healthy elderly women. J Strength Cond Res. 2006;20(4):811-8.

3. Olkoski MM, Tusset D, Matheus SC. Behavior of physiological variable during the hidrogimnastic class. FIEP. 2007;77:593-6.

4. Olkoski MM, Tusset D, Wentz MD, Matheus SC. Comportamento de variáveis fisiológicas durante a aula de hidroginástica com mulheres. Rev bras cineantropom desempenho hum. 2010;12:43-8.

5. Wilmore L, Costill DL. Fisiologia do esporte e do exercício. São Paulo: Manole; 2001. 
6. Denadai BS, Rosas R, Denadai MLDR. Limiar aeróbio e anaeróbio na corrida aquática: comparação com os valores obtidos na corrida em pista. Rev Bras Ativ Fis Saude. 1997;2:23-8.

7. Graef FI, Kruel LFM. Frequência cardíaca e percepção subjetiva do esforço no meio aquático: diferenças em relação ao meio terrestre e aplicações na prescrição do exercício - uma revisão. Rev Bras Med Esporte. 2006;12:221-8.

8. Watenpaugh DE, Pump B, Bie P, Norsk P. Does gender influence human cardiovascular and renal responses to water immersion? J Appl Physiol. 2000;89:621-8.

9. Srámek P, Simeckova M, Janski L, Savlikova J, Vybiral S. Human physiological responses to immersion into water of different temperatures. Eur J Appl Physiol. 2000;81(5):436-42.

10. Graef FI, Tartaruga LAP, Alberton CL, Kruel LFM. Frequência cardíaca em homens imersos em diferentes temperaturas de água. Rev Port Cien Desp. 2005;5:266-73.

11. Kruel LFM, Tartaruga LAP, Alberton CL, Graef F, Petkowicz R. Effects of hydrostatic weight on heart rate during water immersion. IJARE. 2009;3(2):178-85.

12. Craig AB, Dvorak M. Thermal regulation during water immersion. J Appl Physiol. 1966;21(5):1577-85.

13. Arborelius M, Baldlin UI, Lilja B, Lundgren CEG. Hemodynamic changes in man during immersion with the head above water. Aerosp Med. 1972;43:590-8.

14. McArdle WD, Magel JR, Lesmes GR, Pechar CS. Metabolic and cardiovascular adjustment to work in air and water at 18, 25 and 33ํ․ J Appl Physiol. 1976;40(1):85-90.

15. Rennie DW, Di Prampero P, Cerretelli P. Effects of water immersion on cardiac output, heart rate, and stroke volume of man at rest and during exercise. Med Del Sport. 1971;24:223-8.

16. Park KS, Choi JK, Park YS. Cardiovascular regulation during water immersion. J Physiol Appl Hum Scie. 1999;18:233-41.

17. Sheldahl LM, Wann LS, Clifford OS, et al. Effect of central hypervolemia on cardiac performance during exercise. J Appl Physiol. 1984;57(6):1662-7.

18. Kruel LMF, Dias ABC, Silva RC, Picanço PP, Rangel ACB. Frequência cardíaca durante a imersão no meio líquido. Fitness Perform J. 2002;1:46-51.
19. Cassady SL, Nielsen DH. Cardiorespiratory responses of healthy subjects to calisthenics performed on land versus in water. Phys Ther. 1992;72(7):532-8.

20. Alberton CL, Coertjens M, Figueiredo PAP, Kruel LFM. Behavior of oxygen uptake in water exercise performed at different cadences in and out of water. Med Sci Sports Exerc. 2005;37:S103.

21. Johnson BL, Stromme SB, Adamczyk JW, Tennoe KO. Comparison of oxygen uptake and heart rate during exercises on land and in water. Phys Ther. 1977;57(3):273-8.

22. Alberton CL, Olkoski MM, Pinto SS, Becker ME, Kruel LFM. Cardiorespiratory responses of postmenopausal women to different water exercises. IJARE. 2007;1(4):363-72.

23. Moraes EZC, Kruel LFM, Sampedro RMF, Lopes LFD. Metodologia de medida de esforço para exercícios de hidroginástica em diferentes profundidades de água. Kinesis. 2002;27:43-186.

24. Pinto SS, Alberton CL, Becker ME, Olkoski MM, Kruel LFM. Respostas cardiorrespiratórias em exercícios de hidroginástica executados com e sem o uso de equipamento resistivo. Rev port cien desp. 2006;6(3):336-41.

25. Pinto SS, Alberton CL, Figueiredo PAP, Tiggemann CL, Kruel LFM. Respostas de frequência cardíaca, consumo de oxigênio e sensação subjetiva de esforço em um exercício de hidroginástica executado por mulheres em diferentes situações com e sem o equipamento Aquafins ${ }^{\circledR}$. Rev Bras Med Esporte. 2008;14(4):357-36.

26. Pöyhönem T, Keskinen KL, Hautala A, Mälkiä E. Determination of hydrodynamic drag forces and drag coefficients on human leg/foot model during knee exercise. Clin Biomech. 2000;15(4):256-60.

Recebido: $16 / 02 / 2012$ Received: 02/16/2012

Aprovado: 30/09/2012 Approved: 09/30/2012 